\title{
„Mit engagierten Versorgungsprojekten den Herausforderungen der Demografie begegnen"
}

\author{
Anlässlich der Herbsttagung der Deutschen Diabetes-Gesellschaft hat das Unternehmen Berlin- \\ Chemie bereits zum zweiten Mal den SilverStar-Preis verliehen - ein Preis, der gezielt Projekte \\ fördert, welche älteren Diabetikern eine bessere Lebensqualität ermöglichen. Über die Heraus- \\ forderungen der geriatrischen Diabetiker-Betreuung sowie die Besonderheiten der diesjährigen \\ Preisverleihung unterhielt sich SpringerMedizin mit dem SilverStar-Jury-Mitglied Professor \\ Cornel Sieber (re.), Chefarzt der Med. Klinik 2, Universität Nürnberg-Erlangen.
}

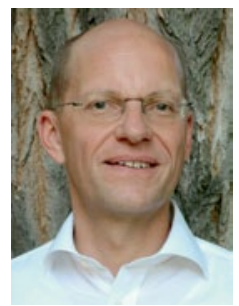

Herr Professor Sieber, welches sind die Herausforderungen in der Diabetologie beim geriatrischen Patienten?

Sieber: In der Geriatrie haben wir es häufig mit Patienten zu tun, die mehrere Krankheiten haben und viele Medikamente einnehmen. Diabetes ist bei diesen Patienten eine der häufigsten Komorbiditäten. In unserer Klinik beträgt das Durchschnittsalter 83 Jahre und ca. 25\% der Patienten haben einen Diabetes.

\section{Priorisierung in der Geriatrie}

Die Herausforderung für den Geriater besteht meist darin, eine gewisse Priorisierung innerhalb der Multimorbidität vorzunehmen und jene Störungen zu behandeln, welche die Funktionalität des Patienten einzuschränken drohen. Der Diabetes nimmt hier einen hohen Stellenwert ein, weil er zu Komplikationen in mehreren Organsystemen führen kann, die den Patienten deutlich behindern. Daher ist es wichtig, durch eine gute Stoffwechselführung den Erhalt des Augenlichtes oder der Nierenfunktion sicherzustellen und Durchblutungsstörungen der Beine oder eine Polyneuropathie zu vermeiden. Letztere kann zu Gangunsicherheit führen und trägt dann dazu bei, dass alte Menschen stürzen. Ein schlecht eingestellter Diabetes ist nicht zuletzt auch ein Hochrisikofaktor für die Entwicklung einer Demenz, deren Häufigkeit mit der Alterung der Bevölkerung deutlich ansteigt.

Können wir denn die Ergebnisse aus randomisierten kontrollierten Studien in der Diabetologie ohne Weiteres auf geriatrische Patienten übertragen?

Sieber: Im Vergleich zu anderen Krankheiten ist die Datenlage beim Diabetes im Alter recht gut. Wir wissen, dass es sich lohnt, den Stoffwechsel des Diabetikers auch im Alter gut einzustellen, wobei man bei den Zielwerten etwas großzügiger ist. Denn im Alter sollten Hypoglykämien aus vielen Gründen unbedingt vermieden werden.

Das Hauptproblem bei den randomisierten Studien für uns Geriater besteht darin, dass in diesen Studien Multimorbidität oft eingeschränkt oder ausgeschlossen wird und sich das Studienkollektiv daher vom Klinikalltag unterscheidet. Wenn ich aber alle Krankheiten eines multimorbiden Menschen einzeln und leitliniengerecht behandele, dann komme ich in Schwierigkeiten. Daher müssen wir, wie gesagt, priorisieren.

Wird der Diabetes zunehmend zu einer geriatrischen Erkrankung?

Sieber: Diabetes nimmt deutlich im Alter zu. Man kann schon sagen, dass er nicht nur eine Volkserkrankung ist, sondern auch eine Alterskrankheit wird.

Wie beurteilen Sie vor diesem Hintergrund das Projekt SilverStar?

Sieber: Mir hat der SilverStar von Anfang

Jetzt für SilverStar 2013

\section{bewerben!}

Der SilverStar Förderpreis wird für 2013 das 3. Mal ausgelobt. Der Förderpreis soll Bewerber und Projekte unterstützen, die sich die praktische Betreuung von älteren Menschen mit Diabetes zur Aufgabe gemacht haben. Ob Gesundheitsprofis, wie Ärzte, Pflegekräfte, Apotheker, oder engagierte Angehörige, Freunde, Nachbarn oder auch Betroffene: Jede Einzelperson, Gruppe oder Organisation kann sich um den SilverStar bewerben. Die Bewerbungsfrist für 2013 endet am 30. Juni.

Nähere Infos unter: www.silverstar-preis.de an sehr gut gefallen, weil hier Projekte gefördert werden, die die Versorgung älterer Menschen mit Diabetes im Fokus haben. Wir haben in Deutschland das Problem, dass die Versorgungsforschung nicht den gleichen Stellenwert hat wie in vielen anderen europäischen Ländern und entsprechend Versorgungstrukturen weniger untersucht und gefördert werden. SilverStar füllt hier eine Lücke, in dem es Projekte auszeichnet, die multidisziplinär sind und im Alltagpraktikabel sind. Damit hat dieser Preis einen visionären Charakter.

\section{Beeindruckende Zahl und Qualität der Versorgungsprojekte}

Sind Sie überrascht, dass sich mehr als 50 Projekte für den Preis beworben haben? Sieber: Absolut, und dies zeigt, dass es im deutschen Gesundheitssystem viele gibt, die sich mit persönlichem und teilweise ehrenamtlichem Engagement bemühen, die Versorgung von älteren Menschen mit Diabetes zu verbessern. Wenn ich mir die Zahl der Bewerbungen und die Qualität der Projekte ansehe, die sich hier beworben haben, dann beeindruckt mich das sehr. Ich glaube, dass wir den Herausforderungen des demografischen Wandels nur mit engagierten Projekten wie diesen begegnen können. Der Preis ist wegweisend, weil er Menschen dazu anregt, sich ebenfalls zu engagieren, wenn sie sehen, dass die Versorgung älterer Diabetiker ein Thema ist, das gefördert und ausgezeichnet wird. Ich bin zuversichtlich, dass auch in den kommenden Jahren wieder viele innovative und kreative Projekte eingereicht werden und die Zahl der Einreichungen sogar noch weiter ansteigen wird.

Interview: Dr. med. Dirk Einecke 